\title{
A major outbreak of conjunctivitis caused by coxsackievirus A24, Réunion, January to April 2015
}

N Marguerite ${ }^{1}$, E Brottet ${ }^{1}$, F Pagès ${ }^{1}$, MC Jaffar-Bandjee ${ }^{2}$, I Schuffenecker ${ }^{3}$, L Josset ${ }^{3}$, P Vilain ${ }^{1}$, L Filleul ${ }^{1}$

1. Santé publique France, French national public health agency, Regional unit (Cire océan Indien), Saint-Denis, Réunion, France.

2. Laboratory of virology, Centre Hospitalier Universitaire, Saint-Denis, Réunion, France

3. National Reference Centre for Enteroviruses, Hospices Civils de Lyon, Bron, France

Correspondence: Nadège Marguerite (nadege.marguerite@ars.sante.fr)

From January to April 2015, Réunion experienced a major outbreak of acute haemorrhagic conjunctivitis (AHC) caused by coxsackievirus A24, which heavily impacted the healthcare system. According to the general practitioners' (GP) sentinel network, the number of medical consultations due to conjunctivitis during this period was estimated at ca 100,000 . This report describes the characteristics of the outbreak, which were obtained through several different yet complementary surveillance systems on the island. These included the network of hospital emergency departments (OSCOUR network), the GPs' sentinel network, an Internet-based population cohort ('Koman i lé') participating in a survey on distinct symptoms including 'red eyes' and the monitoring of eye drop sales. Overall the results of the different surveillance approaches were in good agreement regarding the outbreak dynamic. A peak of patients with conjunctivitis was detected in the first 15 days of March (week 10 and 11), coinciding with increased eye drop sales on the island. Strains recovered from outbreak cases belonged to genotype IV and were most closely related to strains identified in AHC outbreaks in China, Egypt and Japan since 2010. Continued surveillance of AHC in Réunion remains important not only locally, but also because frequent exchanges between the island and mainland France may lead to introduction of this virus in Europe.

\section{Introduction}

Réunion is a French overseas territory located in the South-West Indian Ocean (SWIO). It is an island located between Madagascar and Mauritius, with, in 2013, a population of 840,000 inhabitants. Daily exchanges of goods and people occur between Réunion and other islands of the SWIO, which include the Comoros, Madagascar, Mauritius, Mayotte and the Seychelles. Medical facilities in Réunion are similar to those in mainland France, and there are more than 1,000 general practitioners (GP) distributed throughout the island, as well as four hospitals and six emergency departments (ED).

In tropical countries with high population density, epidemics of viral conjunctivitis occur mainly during the hot and rainy season [1]. They are mostly attributed to adenoviruses and enteroviruses (EV) $[2,3]$. Enteroviruses are ubiquitous pathogens responsible for a large range of infections [3]. There is no specific antiviral treatment. The two main serotypes of EV associated with acute haemorrhagic conjunctivitis (AHC) are enterovirus 70 (EV-70) and coxsackievirus A24 (CV-A24V). CV-A24V was detected for the first time during an outbreak in Singapore in 1970 [4]. The outbreaks of conjunctivitis caused by CV-A24V are particularly explosive, whereby the disease is highly contagious, and has a very short incubation period (12 hours to 3 days), leading to high attack rates [5]. In 2002, an epidemic of AHC due to CV-A24V occurred in South Korea, involving more than a million cases. The virus then spread in worldwide causing a pandemic [3].

Although a large outbreak of conjunctivitis with more than 12,000 cases was detected in 2012 in Mayotte [6], no outbreak of conjunctivitis in Réunion has been described in the 10 years prior to 2015. At the end of January 2015 however, a cluster of cases of conjunctivitis was detected in the west part of the island in the municipality of Saint-Paul, by a surveillance network that monitors data from emergency departments (ED), i.e. the 'Organisation de la surveillance coordonnée des urgences' (OSCOUR). Further investigations revealed the beginning of an outbreak that spread throughout the island.

\section{Methods}

\section{Epidemiological surveillance}

In Réunion, conjunctivitis is monitored routinely by the OSCOUR network [7]. This syndromic surveillance 


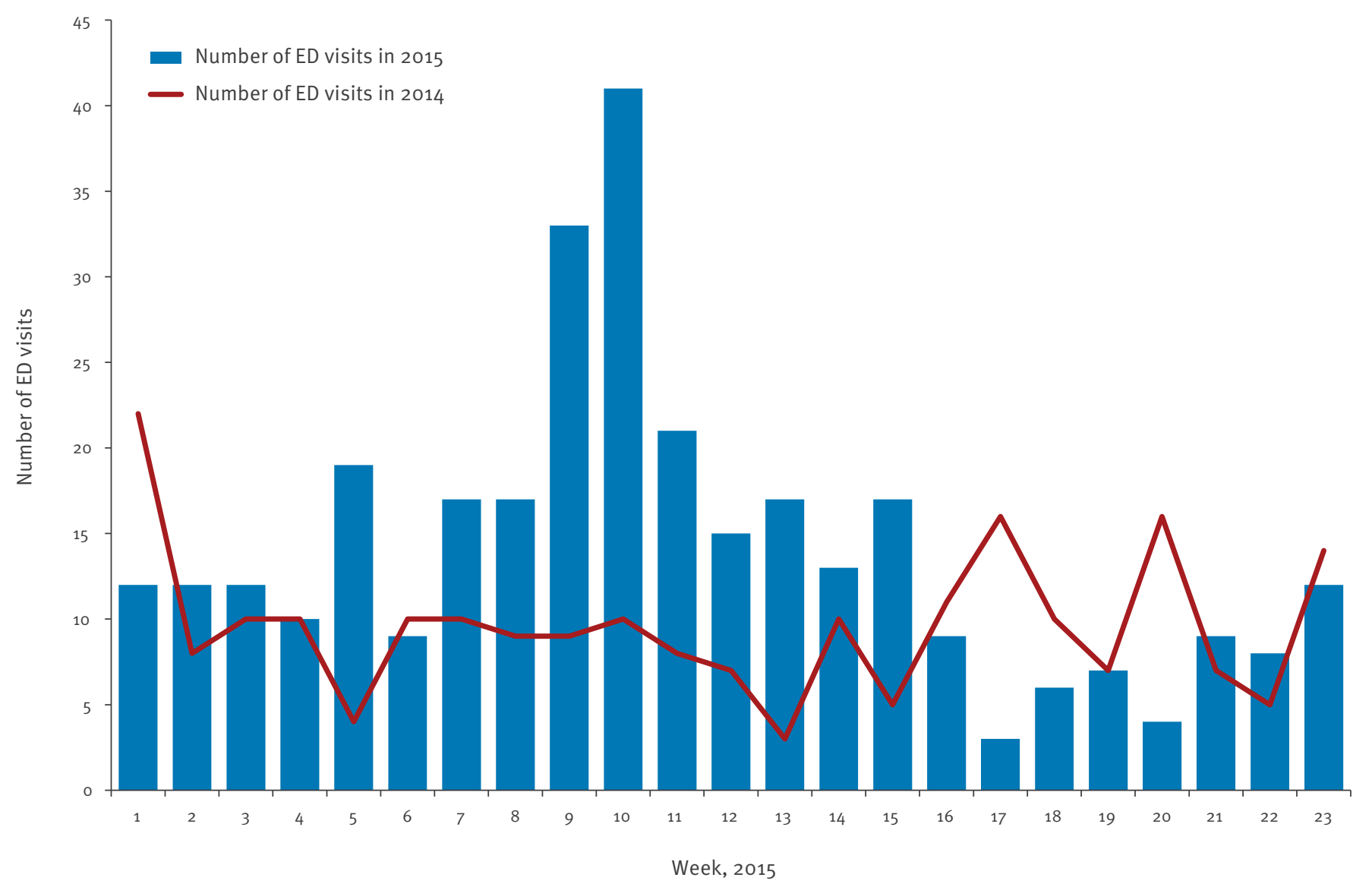

ED: emergency department; InVs: Institut de veille sanitaire (French Institute for Public Health Surveillance); OSCOUR: Organisation de la surveillance coordonnée des urgences.

a For comparison, weekly number of ED visits for conjunctivitis in the same period of the previous year are provided.

system is based on data collected by the six EDs of the island. Data are collected daily directly from patients' computerised medical files that are filled in during medical consultations [8]. For each ED visit, several variables are collected, including patient age, sex, city of residence and the diagnosis. This allows for analysis by syndromic groups, age groups and geographical areas. The diagnosis is categorised according to the $10^{\text {th }}$ revision of the international Classification of Diseases (ICD-10) [9]. Several indicators are routinely monitored, including the number of ED visits for conjunctivitis (ICD-10 codes $\mathrm{B} 30$ and sub-codes, codes $\mathrm{H}_{10}$ and sub-codes, codes $\mathrm{H}_{11}$ and sub-codes). Temporal and spatio-temporal analyses are carried out every day from these data.

On 27 January 2015, data from the OSCOUR network allowed to detect a cluster of conjunctivitis cases in the municipality of Saint-Paul during week 4 (20 to 27 January 2015). The same day, six GPs of this municipality were interviewed by phone and they confirmed an outbreak. On 28 January 2015, after interview, several sentinel GPs reported that the outbreak had spread to the whole island. In this context, from the end of January (week 5) routine surveillance was enhanced by specific surveillance of conjunctivitis using data from sentinel GPs and a sentinel population network.

The sentinel GPs' network is based on 56 volunteer GPs located throughout the island, who report to the regional office of the French Institute for Public Health Surveillance (Cire OI) the weekly number of consultations for acute respiratory infections and acute diarrhoea and the total number of consultations $[10,11]$. These GPs are easily mobilised at the onset of any health event on the island and they can be asked to add specific items to their weekly report. At the beginning of the week 5 , the Cire Ol suggested to the sentinel GPs to report, in addition to usual indicators, the weekly number of consultations for conjunctivitis according to the following case definition: inflammation of the eye with burning sensation or watering eyes 
Estimation of the weekly number of consultations carried out by general practitioners for conjunctivitis, Réunion, 29 December 2014-7 June $2015(\mathrm{n}=$ ca 104,403)

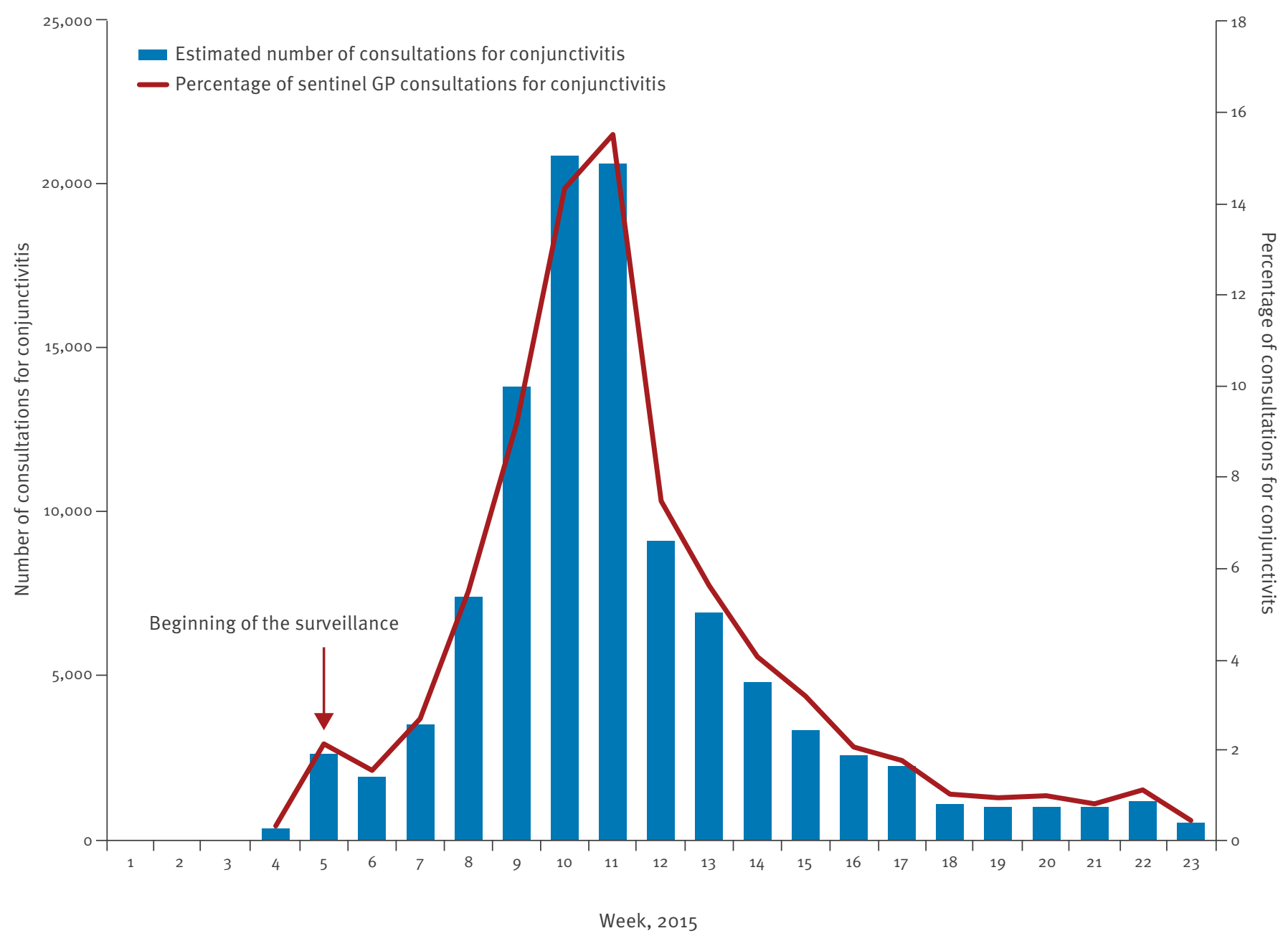

GP: general practitioner.

The estimation of weekly consultations for conjunctivitis $=A /(B / C * D)$ where: $A$ is the weekly number of sentinel general practitioner's $(G P)$ consultations for conjunctivitis; B is the weekly number of sentinel GP's consultations all causes; C is the weekly number of consultations carried out by all GPs and reimbursed by the national health insurance centre of Réunion (CGSS); $D$ is the coverage of CGSS data in general population ( $72 \%)$.

or lacrimal secretion or light sensitivity. To estimate the total number of consultations for conjunctivitis on the whole island, data from the GPs were extrapolated using data from the national health insurance centre of Réunion (Caisse Générale de Sécurité Sociale; CGSS) $[11,12]$. Each week, the CGSS sent by email to Cire OI, the aggregated number of medical consultations and home visits carried out in the prior week (week -1) by GPs and reimbursed by CGSS. The transmitted data concerned $72 \%$ of the population of the island.

Since April 2014, the Cire Ol pilots an experimental surveillance system based on the use of the Internetbased cohort 'Koman i lé'. Individual volunteers aged over 18 years with Internet access and living in Réunion are included. In total, 350 adult volunteers contribute to this surveillance system. They fill in anonymously a short survey every week asking if they had any of 17 symptoms during the previous week [13]. Each week for each symptom, the percentage of participants who presented the symptom compared with all participants who answered the questionnaire is calculated. This allows monitoring trends of different symptoms. Among these symptoms, one is 'red-eyes'.

Data from these three surveillance systems were analysed by the Cire Ol.

Furthermore, to assess the impact on the population, the use of collyrium was monitored in real time. A supplier of some $40 \%(100 / 250)$ of the pharmacies on the island sent to the Cire Ol its data on eye drop sales 


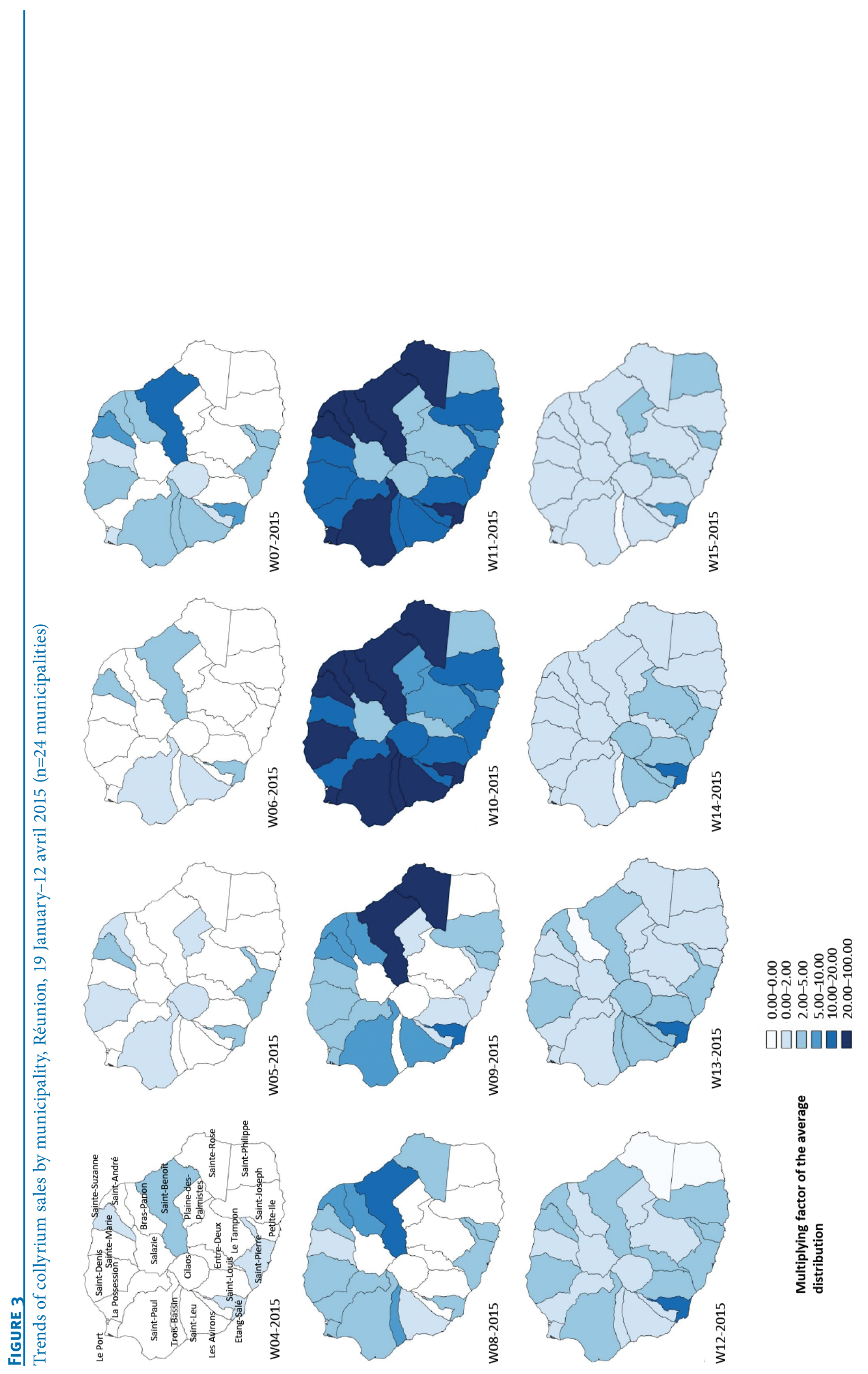


from the beginning of 2015 . For each municipality on the island, collyrium sales for weeks 4 to 15 were compared with the average collyrium sales in weeks 2 and 3 , before the outbreak.

Virological screening of conjunctival specimen In order to determine the aetiology of this outbreak, sentinel GPs were encouraged to collect swabs from conjunctivitis cases. As a first step, twenty-six conjunctivitis specimens were analysed by the hospital laboratory of Saint-Denis using a real-time polymerase chain reaction (RT-PCR) detecting adenoviruses (Adenovirus R-gene, bioMérieux) and a multiplex PCR detecting enteroviruses (Respifinder Smart 22, Pathofinder).

\section{Molecular typing of enterovirus positive} samples and phylogenetic analyses

Among the conjunctivitis specimens testing positive for enteroviruses, ten randomly selected samples were sent to the National Reference Centre for Enteroviruses (Lyon, France) for further characterisation. Partial viral protein 1 ( $\left(\mathrm{P}_{1}\right)$ coding sequences were determined using the Nix method [14] and identified as CV-A24V strains using Basic Local Alignment Search Tool (BLAST) software [15]. For phylogenetic analysis, a nt alignment (330 bp) including nine of the $10 \mathrm{VP}_{1}$ sequences determined from conjunctival specimens (one sequence of shorter length was excluded), thirty selected CV-A24 $V P_{1}$ sequences from clinical strains representative of the genotypes I, II, II and IV and the prototype CV-A24 strain (Joseph) was performed. Genetic distances were calculated with the Tamura-Nei model of evolution. The tree was constructed by the neighbour-joining method using molecular evolutionary genetics analysis (MEGA) 5 and validated using 1,000 bootstrap pseudoreplicates. The nine VP1 sequences determined in this study, and included in the phylogenetic analysis, were deposited in GenBank database (accession numbers: KR399980-KR399986; KR399988; KR478685). Two of those were deposited as part of a complete genome sequence (see below).

Metagenomic sequencing and de novo assembly of consensus sequences

Metagenomic sequencing was attempted on three conjunctival swab specimens. Viral nucleic acid enrichment was performed by a combination of centrifugation (10 min at 6,000 $\mathrm{g}$ ), filtration (at $0.45 \mu \mathrm{m}$ )

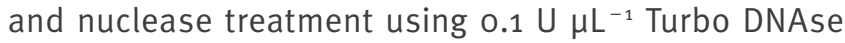
(Life Technologies) as described before [16]. Nucleic acid extraction was then performed with a NucliSens easyMAG magnetic bead system (BioMérieux). cDNA synthesis was performed as described before [17] with some modifications. Reverse transcription was performed with $50 \mathrm{ng}$ of random hexamers (New England Biolabs) and $200 \mathrm{U}$ of SuperScript III reverse transcriptase enzyme (Life Technologies) followed by RNA digestion with $2 \mathrm{U}$ of RNase $\mathrm{H}$ (Life Technologies). After the reverse transcription reaction, second strand synthesis was performed with $5 \mathrm{U}$ Klenow frament (3ם$5 \square$ exo-) (New England Biolabs) and $1 \mu \mathrm{g}$ of random hexamers. cDNA was purified and concentrated using a DNA Clean and Concentrator column (Zymo Research) and the totality of the specimen was used to prepare sequencing libraries using NEBNext Fast DNA Fragmentation and Library Prep Set for Ion Torrent (New England Biolabs) with 12 PCR cycles. The three barcoded libraries were pooled at equimolar concentrations and sequenced on an Ion Torrent PGM instrument (Life Technologies) with an Ion 318 Chip v2. Ion Torrent data was de-multiplexed and quality-trimmed using Ion Torrent Suite software (version 4.4.2). Reads were then assembled using MIRA (v. 4.0.1) [18] and contigs were further merged via V-Fat [19]. Two of the three resulting consensus sequences were deposited into GenBank database (KR399988; KR478685). Consensus sequences were aligned with all complete $\mathrm{CV}$-A24 sequences available in GenBank and potential recombinations were assessed by using the bootscanning method implemented in SimPlot [20].

\section{Results}

\section{Outbreak detection}

At the end of January 2015, the spatio-temporal analysis of data from the OSCOUR network allowed to detect a cluster of conjunctivitis cases in the municipality of Saint-Paul that had occurred during week 4 (20 to 27 January) 2015. GPs in the area, as well as the sentinel GPs in the whole island were interviewed. They confirmed an increase in cases and highlighted that it concerned the whole island. Several practitioners also reported a clinical picture compatible with a highly contagious viral infection.

\section{Outbreak description}

Emergency department visits for conjunctivitis The peak of emergency department visits for conjunctivitis was reached in week 10 (first week of March). The first increase of ED visits in 2015 was visible in week 5 , when the beginning of the epidemic was detected. From week 1 to week 17, a total of 277 ED visits for the disease were recorded on the whole island, against 162 over the same period in 2014 (Figure 1).

The ratio male/female was $1.4(160 / 117)$. The analysis by age group showed that $44 \%(122 / 277)$ of patients who presented to the ED for conjunctivitis were under 20 years of age, and 55\% (152/277) were under 30 years-old (Table). The repartition by age of the patients was not statistically different compared with 2014: $48 \%(78 / 162)$ under 20 years and 58\% (94/162) under 30 years.

\section{Consultations for conjunctivitis of sentinel general practitioners}

The beginning of the outbreak in week 5 was confirmed by the GPs' network. Their reports showed that the outbreak had spread throughout the island in late January and ended in week 17 (end of April) (Figure 2). The outbreak lasted 12 weeks. The epidemic peak was reached 


\section{FIGURE 4}

Phylogenetic analysis, based on coxsackievirus A24 partial viral protein 1 coding sequences, of strains recovered in the outbreak of acute haemorrhagic conjunctivitis, Réunion, 2015

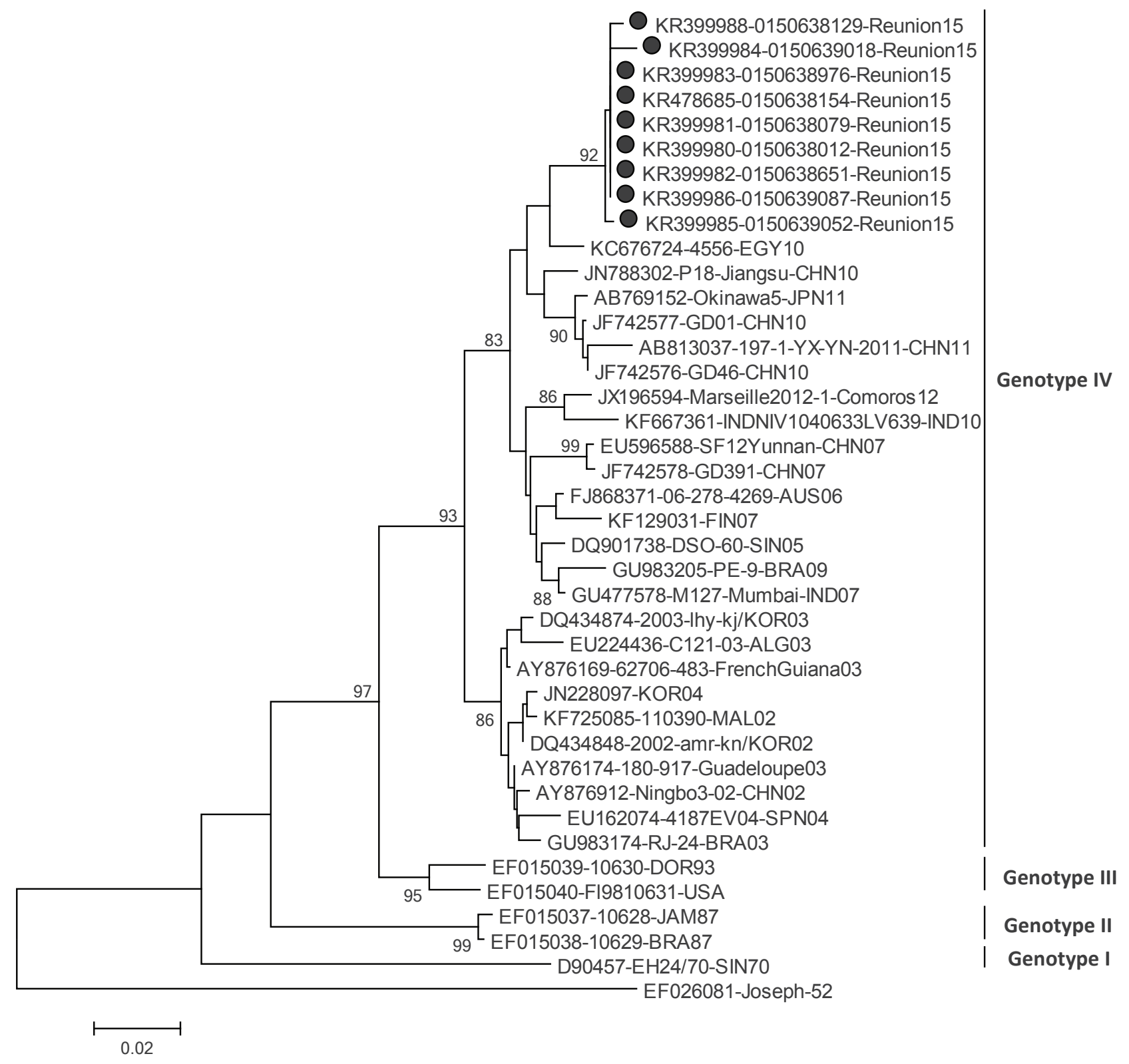

CV-A24v: coxsackievirus A24 variant.

Phylogenetic analysis based on partial VP1 coding sequences ( $330 \mathrm{bp}$ ) of CV-A24V strains. The analysis included nine VP1 sequences determined in this study from conjunctival swab specimens collected during the outbreak in Réunion (shown by a dot), thirty selected sequences from clinical strains representative of the genotypes I, II, II and IV and the prototype CV-A24 Strain (Joseph). Genetic distances were calculated with the Tamura-Nei model of evolution. The tree was constructed by the neighbour-joining method using molecular evolutionary genetics analysis (MEGA) 5 and validated using 1,000 bootstrap pseudo-replicates. The nine VP1 sequences determined in this study and included in the phylogenetic analysis were deposited in GenBank database (accession numbers: KR399980-KR399986; KR399988; KR478685).

in week 11, one week later than in the ED. For each of the weeks 10 and 11 , more than 20,000 consultations for conjunctivitis were estimated, and the whole island was affected. The total number of consultations for conjunctivitis on the whole island during this outbreak was estimated to be ca 100,000. The epidemic curve shows a highly contagious outbreak.
Results from 'Koman i lé'

From week 8 to week 10, the percentage of participants reporting red eyes was between $7 \%(7 / 104)$ in week 8 and $8 \%(8 / 106)$ in weeks 9 and 10 . In contrast this percentage was $3 \%(3 / 108)$ in week 7 . Overall among adults having declared that they had red eyes during this period, $22 \%(5 / 23)$ consulted a GP. 
TABLE

Distribution by age groups of patients visiting the emergency department for conjunctivitis, Réunion, 29 December-26 April $2015(\mathrm{n}=277)$

\begin{tabular}{|l|c|}
\hline Age group (in years) & $\begin{array}{c}\mathrm{n} \text { (\% among emergency } \\
\text { department visits for } \\
\text { conjunctivitis) }\end{array}$ \\
\hline $0-9$ & $97(35)$ \\
\hline $10-19$ & $25(9)$ \\
\hline $20-29$ & $30(11)$ \\
\hline $30-39$ & $36(13)$ \\
\hline $40-49$ & $45(16)$ \\
\hline $50-59$ & $22(8)$ \\
\hline$\geq 60$ & $21(8)$ \\
\hline
\end{tabular}

Data from the supplier

Data from the supplier of the pharmacies showed that the sales of eye drops increased rapidly on the whole island (Figure 3). Sales were multiplied by twenty in 11 municipalities during week 10 and in eight municipalities during week 11. Almost all municipalities of the island were affected. However, coastal municipalities were more impacted than municipalities in the centre of the island, which are located in mountainous areas and less populated.

\section{Laboratory investigations}

Of the twenty-six swabs collected by the sentinel GPs, twenty-three of them tested positive by RT-PCR for enteroviruses and negative for adenoviruses. Ten randomly selected samples were sent to the National Reference Centre for Enteroviruses for further characterisation. CV-A24V belonging to genotype IV was identified in all the samples by sequencing of the VP1 coding region (Figure 4). The $10 \mathrm{VP}_{1}$ partial nt sequences shared a 99.1 to $100 \%$ nt identity and had 95.1 to $97.9 \%$ nt similarity with sequences from genotype IV isolates previously identified in AHC outbreaks in Egypt (2010) [21], Japan (2011) [22], China (2007 and 2010) [23-25], Brazil (2009) [26], India (2007) and Singapore (2005) [27] (Figure 4). They also grouped in a larger cluster containing the $\mathrm{VP}_{1}$ sequence from a genotype IV isolate detected in a traveller returning from Comoros to France in 2012 (Figure 4; accession number JX196594). Two whole genome sequences were generated by a metagenomic approach and showed a 97.2-97.3\% similarity with sequences from Japanese strains detected in 2011 in Okinawa [22]. Similarity plots provided no evidence of recombination (data not shown).

\section{Discussion}

Between January and April 2015, a major outbreak of AHC occurred in Réunion, being the first of such outbreaks ever described on the island. The outbreak was caused by strains of CV-A24V belonging to genotype IV. In a phylogenetic analysis, nt sequences of the outbreak strains grouped with other strains identified since 2002 in AHC outbreaks in Africa, America and Asia. Within this group, the sequences from this study were most closely related to those of recent AHC outbreaks (i.e. China 2010, Egypt 2010 and Japan 2011), which belong to a sub-cluster that might have emerged between 2008 and 2009 [22].

During the same period as the Réunion outbreak, an outbreak of conjunctivitis also occurred in Mauritius, but the only viral aetiology identified was adenovirus. Then, in early March, similar outbreaks began in Madagascar and the Seychelles but no data are available on the causative agents. Three years prior, from February to May 2012, Mayotte had experienced an outbreak of acute conjunctivitis, most likely caused by a CV-A24V closely related to the isolates associated with the Réunion outbreak $[6,28]$.

Because outbreaks of AHC can reappear and due to the frequent exchanges between Réunion and mainland France, there is a recurring risk of further exportation of the virus to France and other European countries through returning travellers. The exchanges between the SWIO islands are also important but surprisingly the two concomitants conjunctivitis outbreaks in Mauritius and Réunion were due to different viruses. This highlights the need for specific laboratory investigations in each island even when similar outbreaks occur in the same period.

In Réunion, information about the outbreak was broadcasted to the population [29], by the local media (radio, television and websites) together with advice on how to avoid infection. For example, following good hygiene practices was recommended, such as washing hands regularly with soap and water, avoid rubbing eyes and using clean water or a disposable tissue to rinse eyes. The first epidemiological report was broadcasted on 30 January 2015 and the last on 30 April 2015. In total, nine weekly epidemiological reports were published.

This study shows the usefulness of several complementary surveillance systems. It also underscores the importance of cooperation between different health professionals, which were quickly mobilised. The different surveillance systems produced consistent results. Indeed, the OSCOUR network, the sentinel GP network and the sentinel population network 'Koman I lé' showed the same outbreak dynamic. Moreover, the most important sales of collyrium occurred in weeks 10 and 11 , which corresponds to epidemic peaks visible by the three other surveillance systems.

Nevertheless, each of these systems presents strengths and limits.

The main strength of the OSCOUR network is that it enabled early detection of the outbreak because the data are collected, sent and analysed on a daily basis. Second, several variables are collected for each 
patient, including age and city of residence, which allows for analysis by age groups, and geographical areas. Moreover, data from the six EDs of the island are transmitted daily since 2009 , so comparisons are possible with previous years. The limitation of the OSCOUR network is that it is not the most appropriate surveillance system for monitoring conjunctivitis because few patients go to ED for this pathology. OSCOUR is able to detect community outbreaks but not to assess their range or to monitor their evolution.

The sentinel GPs' network is particularly suitable for monitoring conjunctivitis, this pathology not usually being severe. Moreover, the data from this network, coupled with those from the national health insurance contributed to estimate the impact of the epidemic in terms of number of consultations and to follow its dynamics. Even if this network is less reactive than the OSCOUR network because the data are transmitted once a week, activation of GPs for the surveillance of conjunctivitis was adequate to monitor the outbreak. Nevertheless, no historical data are available to make comparisons with previous years. As this network saw only people seeking medical care for their conjunctivitis, people using self-medication or using traditional phytotherapy, who are numerous on the island, were not taken in account in the assessment of the outbreak burden. Indeed, the outbreak was probably more important than estimated by the GPs' network.

The sentinel population 'Koman i lé' is a new project directly involving the population, including people not consulting their doctors when they are sick. The weakness is that this system is not representative for the population of Réunion. Indeed, only adults can participate, and the elderly are not much involved because they do not use the Internet much. Moreover, some municipalities are still under-represented. At the current stage of development of 'Koman i lé, it is not possible to extrapolate the results of this system to the general population and to use it to evaluate the real burden of an epidemic. In the future, if the number of participants increases, it will be possible to better monitor health events in the community.

The surveillance based on sales of collyrium provides data stratified by municipalities, shows the spatiotemporal dynamic. However, this monitoring is not exhaustive because the supplier who sends its data does not provide for all pharmacies of the island. Nevertheless, data were congruent with those of the others systems and were sufficient to describe the dynamics of the epidemic in the island.

Despite some weaknesses of the surveillance systems, the analyses of all of these complementary sources of data allowed to describe and characterise the outbreak. Information produced by this surveillance system enabled to health authorities to adapt prevention actions to the dynamic of the outbreak.
Acknowledgements

We acknowledge all the sentinel general practitioners of Réunion, the emergency departments of Réunion, the microbiology laboratory of the University Hospital Centre of Saint-Denis, the National Reference Centre for enterovirus, the National Health Insurance Centre of Réunion, the supplier of pharmacies CERP Réunion SAS, the inhabitants of Réunion who participate in Koman I lé and the Health Agency of Indian Ocean.

\section{Conflict of interest}

None declared.

Authors' contributions

All authors contributed to the interpretation of the results, the revision of the draft manuscript and approved the final version. Nadège Marguerite contributed to the epidemiological analyses and wrote the manuscript. Elise Brottet, Frédéric Pagès, Pascal Vilain and Laurent Filleul contributed to the epidemiological analyses and to the writing of the manuscript. Marie-Christine Jaffar-Bandjee was responsible for the viral laboratory analyses. Isabelle Schuffenecker and Laurence Josset conducted the characterisation of the CVA24V strains, performed the phylogenetic analyses and contributed to the writing of the manuscript.

\section{References}

1. Hierholzer JC, Hilliard KA, Esposito JJ. Serosurvey for "acute hemorrhagic conjunctivitis" virus (Enterovirus 70) antibodies in the Southeastern United States, with review of the literature and some epidemiologic implications.Am J Epidemiol. 1975;102(6):533-44.PMID: 1202955

2. Centers for Disease Control and Prevention (CDC). Notes from the field: acute hemorrhagic conjunctivitis outbreaks caused by coxsackievirus A24V -..- Uganda and southern Sudan, 2010.MMWR Morb Mortal Wkly Rep. 2010;59(32):1024.PMID: 20724969

3. Lévêque $\mathrm{N}$, Huguet $\mathrm{P}$, Norder $\mathrm{H}$, Chomel JJ. Les Enterovirus responsables de conjonctivite aiguë hémorragique.

[Enteroviruses responsible for acute hemorrhagic conjunctivitis]. Med Mal Infect. 2010;40(4):212-8. DOI: 10.1016/j.medmal.2009.09.006 PMID: 19836177

4. Mirkovic RR, Schmidt NJ, Yin-Murphy M, Melnick JL. Enterovirus etiology of the 1970 Singapore epidemic of acute conjunctivitis.Intervirology. 1974;4(2):119-27.PMID: 4217326

5. Heymann DL. Control of communicable diseases manual. 19th Edition. Washington, DC: American Public Health Association;2008. p.746.

6. Lernout T, Maillard O, Boireaux S, Collet L, Filleul L. A large outbreak of conjunctivitis on Mayotte Island, France, February to May 2012.Euro Surveill. 2012;17(23):20192.PMID: 22720741

7. Josseran L, Fouillet A, Caillère N, Brun-Ney D, Ilef D, Brucker G, et al. Assessment of a syndromic surveillance system based on morbidity data: results from the Oscour network during a heat wave. PLoS One. 2010;5(8):e11984. DOI: 10.1371/journal. pone.0011984 PMID: 20711252

8. Vilain P, Filleul L. La surveillance syndromique à la Réunion: un système de surveillance intégré. [Syndromic surveillance in Réunion: An integrated surveillance system]. Bulletin de veille sanitaire océan Indien. 2013;21:912. French. Available from: http://www.invs.sante.fr/ $\mathrm{fr} /$ Publications-et-outils/Bulletin-de-veille-sanitaire/ Tous-les-numeros/Ocean-indien-Reunion-Mayotte/ Bulletin-de-veille-sanitaire-ocean-Indien.-N-21-Septembre-2013

9. World Health Organization (WHO). International statistical classification of diseases and related health problems. 10th revision. Geneva, Switzerland: WHO. [Accessed 2 Jan 2015]. Available from: http://apps.who.int/classifications/icd10/ browse/2016/en

10. Brottet E, Jaffar-Bandjee MC, Rachou E, Polycarpe D, Ristor B, Larrieu S, et al. Sentinel physician's network in Reunion Island: a tool for infectious diseases surveillance. Med Mal Infect. 2015;45(1-2):21-8. DOI: 10.1016/j.medmal.2014.11.004 PMID: 25575412 
11. Brottet E, Vandroux D, Gauzere BA, Antok E, Jaffar-Bandjee $M C$, Michault $A$, et al. Influenza season in Réunion dominated by influenza B virus circulation associated with numerous cases of severe disease, France, 2014. Euro Surveill. 2014;19(39):20916. DOI: 10.2807/1560-7917. ES2014.19.39.20916 PMID: 25306979

12. Vilain P, Caillere N, Larrieu S, Ristor B, Filleul L. Utilisation des données de l'assurance maladie pour la surveillance syndromique à la Réunion. [Using data from health insurance for syndromic surveillance in Réunion]. Bulletin de veille sanitaire océan Indien. 2012;17:10-14. French.

13. "Koman i lé »: une population sentinelle à la Réunion. Présentation du système de surveillance et premiers résultats, avril 2014, La Réunion. ["Koman i lé »: a sentinel population network in Réunion. Introduction to the surveillance system and first results]. Bulletin de veille sanitaire océan Indien. 2014;24:2-5. French. Available from: http://www. invs.sante.fr/fr/Publications-et-outils/Bulletin-de-veillesanitaire/Tous-les-numeros/Ocean-indien-Reunion-Mayotte/ Bulletin-de-veille-sanitaire-ocean-Indien.-N-24-Octobre-2014.

14. Nix WA, Oberste MS, Pallansch MA. Sensitive, seminested PCR amplification of $\mathrm{VP}_{1}$ sequences for direct identification of all enterovirus serotypes from original clinical specimens.J Clin Microbiol. 2006;44(8):2698-704. DOI: 10.1128/JCM.00542-06 PMID: 16891480

15. Altschul SF, Gish W, Miller W, Myers EW, Lipman DJ. Basic local alignment search tool.J Mol Biol. 1990;215(3):403-10. DOl: 10.1016/So022-2836(05)80360-2 PMID: 2231712

16. Hall RJ, Wang J, Todd AK, Bissielo AB, Yen S, Strydom H, et al. Evaluation of rapid and simple techniques for the enrichment of viruses prior to metagenomic virus discovery. J Virol Methods. 2014;195:194-204. DOI: 10.1016/j.jviromet.2013.08.035 PMID: 24036074

17. de Vries M, Deijs M, Canuti M, van Schaik BD, Faria NR, van de Garde MD, et al. A sensitive assay for virus discovery in respiratory clinical samples. PLoS One. 2011;6(1):e16118. DOI: 10.1371/journal.pone.0016118 PMID: 21283679

18. Chevreux B, Wetter T, Suhai S. Genome sequence assembly using trace signals and additional sequence information. In: Proceedings of the German Conference on Bioinformatics: Computer Science and Biology; 1999; Hannover, Germany p.45-56.

19. Charlebois P, Yang X, Newman R, Henn M, Zody M. V-FAT: a post-assembly pipeline for the finishing and annotation of viral genomes; 2012. Available from: http://www. broadinstitute.org/scientific-community/science/projects/ viral-genomics/v-fat

20. Lole KS, Bollinger RC, Paranjape RS, Gadkari D, Kulkarni SS, Novak NG, et al. Full-length human immunodeficiency virus type 1 genomes from subtype C-infected seroconverters in India, with evidence of intersubtype recombination. J Virol. 1999;73(1):152-60.PMID: 9847317

21. Ayoub EA, Shafik CF, Gaynor AM, Mohareb EW, Amin MA, Yassin AS, et al. A molecular investigative approach to an outbreak of acute hemorrhagic conjunctivitis in Egypt, October 2010. Virol J. 2013;10(1):96. DOI: 10.1186/1743-422X-10-96 PMID: 23531270

22. Nidaira M, Kuba Y, Saitoh M, Taira K, Maeshiro N, Mahoe Y, et al. Molecular evolution of $\mathrm{VP}_{3}, \mathrm{VP}_{1}, 3 \mathrm{C}(\mathrm{pro})$ and $3 \mathrm{D}(\mathrm{pol})$ coding regions in coxsackievirus group A type 24 variant isolates from acute hemorrhagic conjunctivitis in 2011 in Okinawa, Japan. Microbiol Immunol. 2014;58(4):227-38. DOI: 10.1111/1348-0421.12141 PMID: 24517637

23. Yan D, Zhu S, Zhang Y, Zhang J, Zhou Y, Xu W. Outbreak of acute hemorrhagic conjunctivitis in Yunnan, People's Republic of China, 2007.Virol J. 2010;7(1):138. DOI: 10.1186/1743-422X-7138 PMID: 20579343

24. Wu B, Oi X, Xu K, Ji H, Zhu Y, Tang F, et al. Genetic characteristics of the coxsackievirus A24 variant causing outbreaks of acute hemorrhagic conjunctivitis in Jiangsu, China, 2010. PLoS One. 2014;9(1):e86883. DOI: 10.1371/ journal.pone.0086883 PMID: 24475191

25. De W, Huanying Z, Hui L, Corina M, Xue G, Leng L, et al. Phylogenetic and molecular characterization of coxsackievirus A24 variant isolates from a 2010 acute hemorrhagic conjunctivitis outbreak in Guangdong, China. Virol J. 2012;9(1):41. DOI: 10.1186/1743-422X-9-41 PMID: 22336176

26. Tavares FN, Campos RM, Burlandy FM, Fontella R, de Melo MM, da Costa EV, et al. Molecular characterization and phylogenetic study of coxsackievirus A24v causing outbreaks of acute hemorrhagic conjunctivitis (AHC) in Brazil. PLoS One. 2011;6(8):e23206. DOI: 10.1371/journal.pone.0023206 PMID: 21858030

27. Ong AE, Dashraath $P$, Lee VJ. Management of enteroviral conjunctivitis outbreaks in the Singapore military in 2005
Southeast Asian J Trop Med Public Health. 2008;39(3):398403.PMID: 18564678

28. Aubry C, Gautret P, Nougairede A, Dussouil AS, Botelho-Nevers E, Zandotti C, et al. 2012 outbreak of acute haemorrhagic conjunctivitis in Indian Ocean Islands: identification of Coxsackievirus A24 in a returned traveller. Euro Surveill. 2012;17(22):20185.

29. Institut de Veille Sanitaire. Point épidémiologique. Epidémie saisonnière de conjonctivite à la Réunion. [Seasonal epidemic of conjunctivitis in Réunion]. Point épidémiologique $\mathrm{n}^{\circ} 18$. Cire Océan Indien;12 Mar 2015. French. Available from: http://www.invs.sante.fr/fr/Publications-et-outils/Pointsepidemiologiques/Tous-les-numeros/Ocean-Indien/2015/ Surveillance-de-la-conjonctivite-a-La-Reunion.-Pointepidemiologique-au-5-mars-2015

\section{License and copyright}

This is an open-access article distributed under the terms of the Creative Commons Attribution (CC BY 4.0) Licence. You may share and adapt the material, but must give appropriate credit to the source, provide a link to the licence, and indicate if changes were made.

This article is copyright of the authors, 2016. 